Pacific Journal of Mathematic 


\section{A COMBINATORIAL PROBLEM IN FINITE FIELDS, I}

\section{Gerald Myerson}

Given a subgroup $G$ of the multiplicative group of a finite field, we investigate the number of representations of an arbitrary field element as a sum of elements, one from each coset of $G$. When $G$ is of small index, the theory of cyclotomy yields exact results. For all other $G$, we obtain good estimates.

This paper formed a portion of the author's doctoral dissertation.

Let $p=2 n+1$ be an odd prime. Consider the $2^{n}$ sums represented by the expression

$$
\pm 1 \pm 2 \pm 3 \pm \cdots \pm n \text {. }
$$

How do these sums distribute themselves among the residue classes modulo $p$ ? The answer is, as uniformly as possible; in fact, if we define $N(a)$ as the number of ways of choosing the signs so that $\pm 1 \pm 2 \pm \cdots \pm n \equiv a(\bmod p)$ then we have

THEOREM 1.

$$
\begin{aligned}
& N(a)=\frac{1}{p}\left(2^{n}-\left(\frac{2}{p}\right)\right) \text { for } a \neq 0(\bmod p), \\
& N(0)=\frac{1}{p}\left(2^{n}-\left(\frac{2}{p}\right)\right)+\left(\frac{2}{p}\right) .
\end{aligned}
$$

Here $(2 / p)$ is the Legendre symbol, that is,

$$
\left(\frac{2}{p}\right)=\left\{\begin{array}{l}
1 \text { if } 2 \text { is a quadratic residue }(\bmod p) \\
-1 \text { if } 2 \text { is not a quadratic residue }(\bmod p) .
\end{array}\right.
$$

Our proof of Theorem 1 will rest on the following lemmas.

LEMma 2. If $a b \not \equiv 0(\bmod p)$ then $N(a)=N(b)$.

Proof. Assume $\sum_{k=1}^{n} u_{k} k \equiv a(\bmod p)$, with $u_{k} \in\{1,-1\}$. Since $a b \not \equiv 0(\bmod p)$ there is a $c$ such that $a c \equiv b(\bmod p)$. Thus we have $\sum_{k=1}^{n} u_{k} c k \equiv b(\bmod p)$. Now for $k=1,2, \cdots, n$, let $c k \equiv u_{k}{ }^{\prime} m_{k}(\bmod p)$, where $1 \leqq m_{k} \leqq n, u_{k}^{\prime} \in\{1,-1\}$; these conditions determine $m_{k}$ and $u_{k}^{\prime}$ uniquely. Thus,

$$
b \equiv \sum_{k=1}^{n} u_{k} c k \equiv \sum_{k=1}^{n} u_{k} u_{k}^{\prime} m_{k} \equiv \sum_{k=1}^{n} u_{k}^{\prime \prime} m_{k}(\bmod p),
$$


with

$$
u_{k}^{\prime \prime} \in\{1,-1\}
$$

Now, the $m_{l}$ are all distinct: if $m_{k}=m_{h}$, then $c k \equiv \pm c h(\bmod p)$, so $k \equiv \pm h(\bmod p)$, so $k=h$ (since $1 \leqq k \leqq n, 1 \leqq h \leqq n)$. Therefore, $b \equiv \sum_{k=1}^{n} u_{k}^{\prime \prime} m_{l k}(\bmod p)$ is a representation of $b$, corresponding to our original representation of $a$. Multiplication by $c^{\prime}$, where $c c^{\prime} \equiv 1$ $(\bmod p)$, returns us to the original representation of $a$. We have established a one-to-one correspondence between the set of representations of $a$ and the set of representations of $b$, and this shows that $N(a)$ is independent of $a$ for $a \neq 0(\bmod p)$.

Now let $N$ denote the common value of $N(a), a \neq 0(\bmod p)$, and note that

$$
N(0)+(p-1) N=2^{n}
$$

by counting the total number of expressions two different ways. We now obtain a second linear relation between $N(0)$ and $N$ through the use of a generating function. Let $\theta$ be any primitive $p$ th root of unity.

\section{LEMMA 3. $\prod_{k=1}^{n}\left(\theta^{k}+\theta^{-k}\right)=\sum_{a=0}^{p-1} N(a) \theta^{a}=N(0)-N$.}

Proof. In expanding the product into a sum of powers of $\theta$ each term is of the form $\theta^{ \pm 1 \pm 2 \pm \cdots \pm n}$. The number of occurrences of $\theta^{a}, 0 \leqq a \leqq p-1$, is therefore the number of choices of signs for which $\pm 1 \pm 2 \pm \cdots \pm n \equiv a(\bmod p)$, which is $N(a)$. This proves the first equality. The second follows from Lemma 2 and the observation that $\sum_{a=0}^{p-1} \theta^{a}=0$.

If we can evaluate $\prod_{k=1}^{n}\left(\theta^{k}+\theta^{-k}\right)$ then we will have two equations for $N(0)$ and $N$.

Lemina 4.

$$
\prod_{k=1}^{n}\left(\theta^{k}+\theta^{-k}\right)=\left(\frac{2}{p}\right)
$$

Proof. $\theta+\theta^{-1}$ is a unit in the ring of integers in $Q(\theta)$; in fact, $\left(\theta+\theta^{-1}\right)\left(\theta+\theta^{5}+\theta^{9}+\cdots+\theta^{2 p-1}\right)=1$. The numbers $\theta^{k}+\theta^{-k}$ are conjugate to $\theta+\theta^{-1}$, thus are also units; hence, $\prod_{k=1}^{n}\left(\theta^{k}+\theta^{-k}\right)$ is a unit. By Lemma 3 this product is a rational integer, hence it must be 1 or -1 . We have 


$$
\begin{aligned}
& \prod_{k=1}^{n}\left(\theta^{k}+\theta^{-k}\right)=N(0)-N, \quad(\text { Lemma } 3) \\
& N(0)-N \equiv N(0)+(p-1) N(\bmod p) \\
& N(0)+(p-1) N=2^{n}, \\
& 2^{n} \equiv\left(\frac{2}{p}\right)(\bmod p) \quad \text { (Euler's criterion). }
\end{aligned}
$$

Thus $\Pi_{k=1}^{n}\left(\theta^{k}+\theta^{-k}\right) \equiv(2 / p)(\bmod p)$; but since the product must equal 1 or -1 , it follows that $\prod_{k=1}^{n}\left(\theta^{k}+\theta^{-k}\right)=(2 / p)$.

Proof of Theorem 1. We now have two linear equations in $N(0)$ and $N$;

$$
\begin{aligned}
& N(0)+(p-1) N=2^{n}, \\
& N(0)-N=\left(\frac{2}{p}\right),
\end{aligned}
$$

where the second equation is a consequence of Lemmas 3 and 4 . Simultaneous solution of these equations yields Theorem 1 .

We now present a generalization of the problem solved above; the remainder of this paper is an attempt to solve the generalized problem. We fix the following notation: $e$ and $f$ are positive integers such that ef $+1=q=p^{\alpha}$ is a prime power, and $\boldsymbol{F}_{q}$ is the field of $q$ elements. The multiplicative group of units of $\boldsymbol{F}_{q}$, denoted $\boldsymbol{F}_{q}^{x}$, is generated by the primitive element $g$. The subgroup $G$, consisting of all the eth powers in $\boldsymbol{F}_{q}^{x}$, is generated by $g^{e}$. The cosets of $G$ in $\boldsymbol{F}_{q}^{x}$ are denoted and defined by $G_{k}=g^{k} G, k=0,1, \cdots$, $e-1$. In particular, $G_{0}=G$. For each $x \in F_{q}$ define $N(x)$ to be the number of solutions of $\sum_{k=0}^{e-1} s_{k}=x$, with $s_{k} \in G_{k}$; that is, $N(x)$ is the number of representations of $x$ as a sum of elements, taking precisely one from each coset. $N(x)$ depends, of course, not only on $x$ but on $e$ and $f$ as well; it is, however, independent of the choice of the generator for $\boldsymbol{F}_{q}^{x}$.

With this notation, our problem is, find $N(x)$.

We note that the case $e=(p-1) / 2, f=2$, where $p$ is prime, is our original problem; if $e=(p-1) / 2$ then $g^{e}=-1, G=\{1,-1\}$, and the cosets of $G$ are the sets $\{k,-k\}, k=1,2, \cdots,(p-1) / 2$.

We now try to solve our new problem by following the solution of the old one. We first note that if $s_{k} \in G_{k}$ and $s_{h} \in G_{h}$ then $s_{k}^{-1} \in$ $G_{-k}$ and $s_{k} s_{h} \in G_{k+h}$, where the subscripts are to be reduced $\bmod e$.

LEMMA 5. If $x y \neq 0$, then $N(x)=N(y)$. 
Proof. Assume $\sum_{k=1}^{e-1} s_{k}=x, s_{k} \in G_{k}$. Since $x y \neq 0$ there is a $z \in \boldsymbol{F}_{q}^{x}$ such that $x z=y$. Thus, $\sum_{k=0}^{e-1} z s_{k}=y$. But multiplication by $z$ merely permutes the cosets $G_{k}$, so this gives a representation of $y$. Multiplication by $z^{\prime}$, where $z z^{\prime}=1$, returns us to the original representation of $x$, so we have a one-one correspondence between the two sets of representations. that

Now let $N$ denote the common value of $N(x), x \neq 0$, and note

$$
N(0)+(q-1) N=f^{e},
$$

by counting the number of sums $\sum_{k=0}^{e-1} s_{k}, s_{k} \in G_{k}$, in two different ways.

To generalize Lemma 3 we need an analogue for the expressions $\theta^{k}+\theta^{-k}$. Letting $\theta$ be a primitive complex $p$ th root of unity we define the periods $\eta_{k}=\sum_{x \in G_{k}} \theta^{T r x}, k=0,1, \cdots, e-1$. Here $\operatorname{Tr}$ is the trace map, $\operatorname{Tr}: \boldsymbol{F}_{q} \rightarrow \boldsymbol{F}_{p}$; the elements of $\boldsymbol{F}_{p} \simeq Z / p Z$ are identified with representatives of the cosets of $p Z$ in $Z$; the value of $\theta^{T r x}$ is independent of the choice of representative since $\theta^{p}=1$. We note that $\eta_{k}$ depends on the parameters $e$ and $f$, and also on $g$ :a different choice of $g$ would permute the $\eta_{k}$ among themselves. Note that in the case $q=p$ we can simply define $\eta_{k}=\sum_{a \in G_{k}} \theta^{a}, k=0,1, \cdots$, $e-1$. In particular, if $f=2$ the periods are seen upon renumbering to be the numbers $\eta_{k}=\theta^{k}+\theta^{-k}$ of our previous discussion.

LEMMA 6. $\prod_{k=0}^{c-1} \eta_{k}=\sum_{x \in F_{q}} N(x) \theta^{T r x}=N(0)-N$.

Proof. In expanding the product into a sum of powers of $\theta$ each term is of the form, $\theta^{T r\left(s_{1}+s_{2}+\ldots+s_{e-1}\right)}, s_{l k} \in G_{k}$. The number of occurrences of $\theta^{T r x}$ is therefore the number of representations of $x$ as $\sum_{k=0}^{e-1} s_{k}, s_{k} \in G_{k}$, which is $N(x)$. This proves the first equality. The second follows from Lemma 5 and the observation that

$$
\sum_{x \in \boldsymbol{F}_{q}} \theta^{T r x}=0 \text {. }
$$

Lemma 6 gives a linear relation between $N(0)$ and $N$ which, together with (1), can be used to evaluate $N(0)$ and $N$ if we can evaluate $\prod_{k=0}^{e-1} \eta_{k}$. For fixed values of $e$, it is often possible to obtain formulas for $\prod_{k=0}^{e-1} \eta_{k}$ using the theory of cyclotomy.

In the next section, we give the definitions and quote the theorems we need from cyclotomy. The reader is referred to [7] for a detailed exposition with proofs.

Cyclotomy. We begin by defining the cyclotomic constants. 
Definition. The cyclotomic constant $(k, h)$ is the number of elements $s \in G_{k}$ such that $1+s \in G_{h}$.

The constants $(k, h)$ depend on our parameters $e$ and $f$; also, a different choice of generator $g$, by permuting the cosets $G_{k}$, will permute the constants $(k, h)$. Their importance in the problem under consideration stems from the next two propositions.

Proposition 7. $\eta_{0} \eta_{k}=\sum_{h=0}^{e-1}(k, h) \eta_{h}+f n_{k}$, where $n_{k}$ is defined by

$$
\begin{aligned}
& n_{0}=1 \text { if } f \text { is even, } \\
& n_{0}=1 \text { if } p=2, \\
& n_{e / 2}=1 \text { if } f \text { and } p \text { are odd, } \\
& n_{k}=0 \text { in all other cases. }
\end{aligned}
$$

Proposition 8. $\eta_{m} \eta_{m+k}=\sum_{h=0}^{e-1}(k, h) \eta_{m+h}+f n_{k}$, where the subscripts are to be interpreted modulo $e$.

Repeated applications of Propositions 7 and 8 will enable us to evaluate $\Pi \eta_{k}$, provided we know the constants $(k, h)$.

The constants are given, in the cases $e=2,3$, and 4 , by the following theorems.

Proposition 9. (Dickson [3, p. 48]). Assume $e=2$.

If $f$ is even, the cyclotomic matrix $M^{(2)}$ is given by $M^{(2)}=$ $\left(\begin{array}{ll}A & B \\ B & B\end{array}\right)$, where $4 A=q-5,4 B=q-1$.

If $f$ is odd, $M^{(2)}=\left(\begin{array}{ll}A & B \\ A & A\end{array}\right)$, where $4 A=q-3,4 B=q+1$.

Proposition 10. (Storer [7, p. 35]). Let $e=3$. Let $c$ and $d$ be defined by $4 q=c^{2}+27 d^{2}, c \equiv 1(\bmod 3)$, and, if $p \equiv 1(\bmod 3)$, then $(c, p)=1$; these restrictions determine $c$ uniquely, and $d u p$ to sign. Then

$$
M^{(3)}=\left(\begin{array}{lll}
A & B & C \\
B & C & D \\
C & D & B
\end{array}\right), \text { where } \begin{aligned}
& 9 A=q-8+c \\
& 18 B=2 q-4-c-9 d, \\
& 18 C=2 q-4-c+9 d \\
& 9 D=q+1+c
\end{aligned}
$$

Proposition 11. (Storer [7, pp. 48, 51]). Let $e=4$. Let $s$ and $t$ be defined by $q=s^{2}+4 t^{2}, s \equiv 1(\bmod 4)$, and, if $p \equiv 1(\bmod 4)$, then $(s, p)=1$; these restrictions determine $s$ uniquely, and $t$ up to sign. 
If $f$ is even, then

$$
M^{(4)}=\left(\begin{array}{llll}
A & B & C & D \\
B & D & E & E \\
C & E & C & E \\
D & E & E & B
\end{array}\right) \quad \text { where } \quad \begin{aligned}
& 16 A=q-11-6 s, \\
& 16 B=q-3+2 s+8 t, \\
& 16 D=q-3+2 s, \\
& 16 E=q+1-2 s .
\end{aligned}
$$

If $f$ is odd, then

$$
M^{(4)}=\left(\begin{array}{llll}
A & B & C & D \\
E & E & B & D \\
A & E & A & E \\
E & D & B & E
\end{array}\right) \text { where } \begin{aligned}
& 16 A=q-7+2 s, \\
& 16 B=q+1+2 s+8 t, \\
& 16 D=q+1-6 s, \\
& 16 E=q-3-2 s .
\end{aligned}
$$

Solutions in the cases $e=2,3,4$.

We can now evaluate $\Pi \eta_{k}, N(0)$, and $N$ in the cases $e=2,3,4$.

THEOREM 12. Let $e=2$. If $f$ is even, then

$$
\eta_{0} \eta_{1}=-\frac{q-1}{4}, N(0)=0, N=\frac{q-1}{4} .
$$

If $f$ is odd, then

$$
\eta_{0} \eta_{1}=\frac{q+1}{4}, N(0)=\frac{q-1}{2}, \quad N=\frac{q-3}{4} .
$$

THEOREM 13. Let $e=3$. Let $c$ be defined by $4 q=c^{2}+27 d^{2}$, $c \equiv 1(\bmod 3)$, and, if $p \equiv 1(\bmod 3)$, then $(c, p)=1$. Then

$$
\begin{aligned}
\eta_{0} \eta_{1} \eta_{2} & =\frac{1}{27}((c+3) q-1), \\
N(0) & =\frac{1}{27}(q+1+c)(q-1), \\
N & =\frac{1}{27}\left(q^{2}-3 q-c\right) .
\end{aligned}
$$

Theorem 14. Let $e=4$. Let $s$ be defined by $q=s^{2}+4 t^{2}, s \equiv$ $1(\bmod 4)$, and, if $p \equiv 1(\bmod 4)$, then $(s, p)=1$. If $f$ is even, then

$$
\begin{aligned}
\eta_{0} \eta_{1} \eta_{2} \eta_{3} & =\frac{1}{256}\left(q^{2}-\left(4 s^{2}-8 s+6\right) q+1\right)=\frac{1}{256}\left((q-1)^{2}-4 q(s-1)^{2}\right), \\
N(0) & =\frac{1}{256}(q-1)(q-3+2 s)(q+1-2 s),
\end{aligned}
$$




$$
N=\frac{1}{256}\left(q^{3}-4 q^{2}+5 q+4 s^{2}-8 s+2\right) .
$$

If $f$ is odd, then

$$
\begin{aligned}
\eta_{0} \eta_{1} \eta_{2} \eta_{3} & =\frac{1}{256}\left(9 q^{2}-\left(4 s^{2}-8 s-2\right) q+1\right)=\frac{1}{256}\left((3 q+1)^{2}-4 q(s-1)^{2}\right), \\
N(0) & =\frac{1}{256}(q-1)(q+5-2 s)(q+1+2 s), \\
N & =\frac{1}{256}\left(q^{3}-4 q^{2}-3 q+4 s^{2}-8 s-6\right) .
\end{aligned}
$$

Proof. Straightforward calculation yields the results on $\Pi \eta_{k}$. We present the case $e=3$ as an example.

By Propositions 7 and 10, we have $\eta_{0} \eta_{1}=B \eta_{0}+C \eta_{1}+D \eta_{2}$, whence

$$
\begin{aligned}
\left(\eta_{0} \eta_{1}\right) \eta_{2} & =B\left(\eta_{0} \eta_{2}\right)+C\left(\eta_{1} \eta_{2}\right)+D\left(\eta_{2}\right)^{2} \\
& =B\left(C \eta_{0}+D \eta_{1}+B \eta_{2}\right)+C\left(D \eta_{0}+B \eta_{1}+C \eta_{2}\right)+D\left(B \eta_{0}+C \eta_{1}+A \eta_{2}+f\right) \\
& =(B C+C D+B D) \eta_{0}+(B D+B C+C D) \eta_{1}+\left(B^{2}+C^{2}+A D\right) \eta_{2}+f D .
\end{aligned}
$$

Substituting for $A, B, C$, and $D$ the values given in Proposition 10, and simplifying via $4 q=c^{2}+27 d^{2}$, we find

$$
\begin{aligned}
27 \eta_{0} \eta_{1} \eta_{2} & =\left(q^{2}-3 q-c\right)\left(\eta_{0}+\eta_{1}+\eta_{2}\right)+\left(q^{2}-1+c q-c\right) \\
& =-\left(q^{2}-3 q-c\right)+\left(q^{2}-1+c q-c\right) \\
& =(c+3) q-1 .
\end{aligned}
$$

The results an $N(0)$ and $N$ then follow from the simultaneous solution of

$$
\begin{aligned}
& N(0)+(q-1) N=f^{e}, \\
& N(0)-N=\prod_{k=0}^{e-1} \eta_{k} .
\end{aligned}
$$

Some special results and some approximations. We present two results of a more specialized nature.

THEOREM 15. If $q$ and $f$ are both odd then $N(0)>N$.

Proof. If $q$ and $f$ are both odd then $-1 \in G_{e / 2}$. Thus for any $k, 0 \leqq k<e / 2, x \in G_{k}$ if and only if $-x \in G_{k+e / 2}$. Then

$$
\eta_{k+e / 2}=\sum_{x \in G_{k+e / 2}} \theta^{T r x}=\sum_{x \in G_{k}} \theta^{T r(-x)}=\sum_{x \in G_{k}} \theta^{-T r x}=\bar{\eta}_{k},
$$

where the overbar indicates complex conjugation. It follows that 


$$
\prod_{k=0}^{e-1} \eta_{k}=\prod_{k=0}^{e / 2-1} \eta_{k} \bar{\eta}_{k}=\prod_{k=0}^{e / 2-1}\left|\eta_{k}\right|^{2}>0 .
$$

But by Lemma $6, N(0)=N+\prod_{k=0}^{e-1} \eta_{k}$.

THeOREm 16. Let $e=4$. If $q-1$ is a square, then $N(0)-N$ is a square.

Proof. By hypothesis, $q=1+4 t^{2}$ : thus, we can take $s=1$ in Theorem 14. If $f$ is even then

$$
N(0)-N=\prod_{k=0}^{3} \eta_{k}=\left(\frac{q-1}{16}\right)^{2} ;
$$

if $f$ is odd then

$$
N(0)-N=\prod_{k=0}^{3} \eta_{k}=\left(\frac{3 q+1}{16}\right)^{2} .
$$

Estimates for $\Pi \eta_{k}$ and $N(x)$. Cyclotomy for $e>4$ has been of continuing interest to mathematicians. The reader is referred to [2] for the cases $e=5,6$, and 8; also to [9], [10], [4], [8], [1], and [5], for the cases $e=10,12,14,16,18$, and 20, respectively. In each of these only the case $q=p$ is discussed. When the problems of cyclotomy have been solved for a given value of $e$, the methods of the proof of Theorem 13 will evaluate $\Pi \eta_{k}$ - see, e.g., [6], for the case $e=5, q=p$. The computations involved are ghastly, as the reader can convince himself by inspecting the references cited above. The author feels that the importance of finding exact expressions for $N$ and $N(0)$ is not sufficient to justify performing these computations. We present instead approximations to $N$ and $N(0)$, based upon a lemma from cyclotomy.

LEMma 17. (a) If either $f$ or $p$ is even, then

$$
\sum_{k=0}^{e-1} \eta_{k}^{2}=q-f
$$

(b) If $f$ and $p$ are both odd, then

$$
\sum_{k=0}^{e-1} \eta_{k} \eta_{k+e / 2}=q-f
$$

Proof. These are both special cases of Lemma 9 in [7].

LEMma 18. (a) If either $f$ or $p$ is even then $\eta_{k}$ is real, $k=$ $0,1, \cdots, e-1$.

(b) If $f$ and $p$ are both odd then $\eta_{k} \eta_{k+e / 2}$ is real and positive, 
$k=0,1, \cdots, e-1$.

Proof. (a) If $f$ is even then $-1 \in G_{0}$. Thus if $x \in G_{k}$ then $-x \in G_{k}$, and $x \neq-x$. Hence, if $\theta^{\operatorname{Trx}}$ appears in $\eta_{k}$, so does $\theta^{\operatorname{Tr}(-x)}=\theta^{-T r x}$. Thus, $\eta_{k}$ is real. If $p$ is even then $p=2$. Thus $\theta=-1$ and $\eta_{k}$ is real.

(b) This was shown in the proof of Theorem 15.

THEOREM 19. $\left|\prod_{k=0}^{e-1} \eta_{k}\right| \leqq((q-f) / e)^{e / 2} ;\left|N(0)-f^{e} / q\right| \leqq((q-f) / e)^{e / 2} ;$ $\left|N-f^{e} / q\right| \leqq q^{-1}((q-f) / e)^{e / q}$.

Proof. If either $f$ or $p$ is even then $\sum_{k=0}^{e-1} \eta_{k}^{2}=q-f$. If both $f$ and $p$ are odd then $\sum_{k=0}^{e-1} \eta_{k} \eta_{k+e / 2}=q-f$. In either case we may, by Lemma 18, apply the inequality of the arithmetic and geometric means. We o.btain $\prod_{k=0}^{e-1} \eta_{k}^{2} \leqq((q-f) / e)^{e}$, or $\left|\prod_{k=0}^{e-1} \eta_{k}\right| \leqq((q-f) / e)^{e / 2}$.

The other two inequalities follow from the first and from the relations $N(0)+(q-1) N=f^{e}, N(0)-N=\prod_{k=0}^{e-1} \eta_{k}$.

The reader is encouraged to compare the approximations of Theorem 19 with the exact results of Theorems 12,13, 14 bearing in mind that $c$ in Theorem 13 and $s$ in Theorem 14 can be as large as $2 \sqrt{q}$ or $\sqrt{q}$, respectively. The approximations are seen to be quite sharp.

The problem of evaluating $\Pi \eta_{k}$ as $q$ varies with $f$, rather than $e$, held fixed requires very different methods from those of Theorems 12,13 , and 14 . We treat this problem in [11].

\section{REFERENCES}

1. L. D. Baumert and H. Fredricksen, The cyclotomic numbers of order eighteen with applications to difference sets, Math. Comp., 21 (1967), 204-219.

2. L. E. Dickson, Cyclotomy, higher congruences, and Waring's problem, Amer. J. Math., 57 (1935), 391-424.

3. - Linear Groups, Dover 1958.

4. J. B. Muskat, The cyclotomic numbers of order fourteen, Acta Arithmetica, 11 (1965/6), 263-279.

5. J. B. Muskat and A. L. Whiteman, The cyclotornic numbers of order twenty, Acta Arith. 17 (1970), 185-216.

6. A. R. Rajwade, The period equation for primes $p$ congruent to 1 (Mod 5), Proc. Camb. Phil. Soc., 69 (1971), 153-155.

7. T. Storer, Cyclotomy and Difference Sets, Markham 1967.

8. A. L. Whiteman, The cyclotomic numbers of order sixteen, Trans. Amer. Math. Soc., 86 (1957), 401-413.

9. —— The cyclotomic numbers of order ten, Proc. Symp. Appl. Math., 10, 95-111.

10. - The cyclotomic numbers of order twelve, Acta Arith., 6 (1960), 53-76.

11. G. Myerson, A combinatorial problem in finite fields, II, to appear in Quarterly J. Math.

Received April 1, 1978.

SUNY

Buffalo, NY 14214 



\section{PACIFIC JOURNAL OF MATHEMATICS}

\section{EDITORS}

DoNALD BABBITT (Managing Editor)

University of California

Los Angeles, California 90024

HUGo Rossi

University of Utah

Salt Lake City, UT 84112

C. C. MOORE and ANDREW OGG

University of California

Berkeley, CA 94720
J. DUGUNDJI

Department of Mathematics University of Southern Californı Los Angeles, California 90007

R. Finn and J. Milgram Stanford University Stanford, California 94305

\section{ASSOCIATE EDITORS}

E. F. BECKENBACH

B. H. Neumann
F. WOLF

K. YOSHIDA

\section{SUPPORTING INSTITUTIONS}

UNIVERSITY OF BRITISH COLUMBIA CALIFORNIA INSTITUTE OF TECHNOLOGY UNIVERSITY OF CALIFORNIA MONTANA STATE UNIVERSITY UNIVERSITY OF NEVADA, RENO NEW MEXICO STATE UNIVERSITY OREGON STATE UNIVERSITY UNIVERSITY OF OREGON
UNIVERSITY OF SOUTHERN CALIFORNIA STANFORD UNIVERSITY UNIVERSITY OF HAWAII UNIVERSITY OF TOKYO UNIVERSITY OF UTAH WASHINGTON STATE UNIVERSITY UNIVERSITY OF WASHINGTON 


\section{Pacific Journal of Mathematics}

Vol. 82, No. $1 \quad$ January, 1979

Werner Bäni, Subspaces of positive definite inner product spaces of countable dimension ...................................... 1

Marilyn Breen, The dimension of the kernel of a planar set..............

Kenneth Alfred Byrd, Right self-injective rings whose essential right ideals

are two-sided

Patrick Cousot and Radhia Cousot, Constructive versions of Tarski's fixed

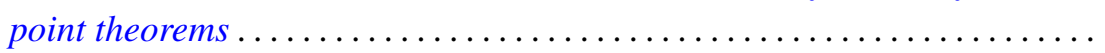

Ralph S. Freese, William A. Lampe and Walter Fuller Taylor, Congruence lattices of algebras of fixed similarity type. $I \ldots \ldots \ldots \ldots \ldots \ldots \ldots$

Cameron Gordon and Richard A. Litherland, On a theorem of Murasugi .....

Mauricio A. Gutiérrez, Concordance and homotopy. I. Fundamental

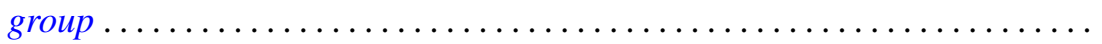

Richard I. Hartley, Metabelian representations of knot groups .............

Ted Hurley, Intersections of terms of polycentral series of free groups and free

Lie algebras ........................................

Roy Andrew Johnson, Some relationships between measures ............ 117

Oldřich Kowalski, On unitary automorphisms of solvable Lie algebras .......

Kee Yuen Lam, $K O$-equivalences and existence of nonsingular bilinear

maps...................................................

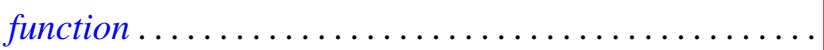

Robert A. Messer and Alden H. Wright, Embedding open 3-manifolds in compact 3-manifolds ............................

Gerald Ira Myerson, A combinatorial problem in finite fields. I . .

James Nelson, Jr. and Mohan S. Putcha, Word equations in a band of paths.

Baburao Govindrao Pachpatte and S. M. Singare, Discrete generalized Gronwall inequalities in three independent variables . .

William Lindall Paschke and Norberto Salinas, $C^{*}$-algebras associated with free products of groups ........................

Bruce Reznick, Banach spaces with polynomial norms ....

David Rusin, What is the probability that two elements of a finite group

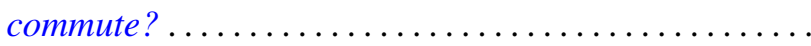

M. Shafii-Mousavi and Zbigniew Zielezny, On hypoelliptic differential operators of constant strength ...

Joseph Gail Stampfli, On selfadjoint derivation ranges .... . . .

Robert Charles Thompson, The case of equality in the matrix-valued triangle

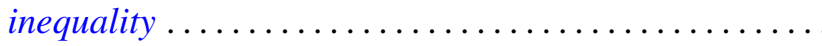

Marie Angela Vitulli, The obstruction of the formal moduli space in the negatively graded case. 